Article

\title{
DFT Investigation on the Electronic, Magnetic, Mechanical Properties and Strain Effects of the Quaternary Compound $\mathrm{Cu}_{2} \mathrm{FeSnS}_{4}$
}

\author{
Jing Bai ${ }^{\dagger}$, Jiaying $\mathrm{Ji}^{\dagger}$, Liyu Hao ${ }^{\dagger}$, Tie Yang ${ }^{\circledR}$ and Xingwen Tan * \\ School of Physical Science and Technology, Southwest University, Chongqing 400715, China; \\ bai123jing@126.com (J.B.); jiaying_ji_1999@foxmail.com (J.J.); hly87411336@email.swu.edu.cn (L.H.); \\ yangtie@swu.edu.cn (T.Y.) \\ * Correspondence: tanxingw@swu.edu.cn \\ + Authors contributed equally.
}

Received: 27 May 2020; Accepted: 10 June 2020; Published: 15 June 2020

\begin{abstract}
The electronic, magnetic and mechanical properties of the quaternary compound $\mathrm{Cu}_{2} \mathrm{FeSnS}_{4}$ have been investigated with first principle calculations. Its half-metallicity has been identified with spin polarized band structures and its magnetic origination is caused by the strong spin splitting effect in the $d$ orbitals of Fe atoms. The total magnetic moment of $4 \mu_{\mathrm{B}}$ is mainly contributed by the Fe atoms and the spatial distribution of the magnetic spin density and charge density difference have also been examined. Moreover, several mechanical properties of $\mathrm{Cu}_{2} \mathrm{FeSnS}_{4}$ have been derived and its mechanical stability is also verified. The directional dependent Young's modulus exhibits relatively small anisotropy yet the shear modulus shows strong directional anisotropy. At last, the tetragonal strain effects have been evaluated and their impact on the electronic and magnetic properties are provided. Results show the total magnetic moment stays almost unchanged while the half-metallicity can only be maintained under relatively small variations for both strains. This study can provide comprehensive information about the various properties of $\mathrm{Cu}_{2} \mathrm{FeSnS}_{4}$ compound and serve as a helpful reference for its future applications.
\end{abstract}

Keywords: first principles calculation; electronic band structure; half-metallicity; strain condition; quaternary compounds

\section{Introduction}

During recent years, the family of half-metallic ferromagnets have aroused strong research interest in the field of material science and condensed matter physics, especially for spintronics and magnetoelectronics [1-6]. Half-metallicity is characterized by the spin polarized band structures with a band gap in one spin direction, similar to semiconductors, and no gap in the other spin direction, similar to metals. A great deal of effort has been dedicated to this material family, both experimental characterizations and theoretical calculations, and, except the intrinsic half-metallicity, various other interesting properties have been found, such as thermoelectricity [7-10], superconductivity [11-13], topological insulativity [14,15], magnetic shape memory [16-21] and so on. Research work is ongoing and devoted to further enhancing their performance or searching for new applications.

In different materials, half-metallic ferromagnets are mostly found in ternary and quaternary compounds. For example, many full Heusler and quaternary Heusler materials have been confirmed to show such a property [22-33]. Compared with ternary materials, quaternary ones are more promising because a larger compositional flexibility can be realized [34-44]. A large and extensively studied quaternary material group is the $\mathrm{A}_{2}^{\mathrm{I}} \mathrm{B}^{\mathrm{II}} \mathrm{C}^{\mathrm{IV}} \mathrm{D}_{4}^{\mathrm{VI}}$ compounds [45-49], in which $\mathrm{A}^{\mathrm{I}}$ is $\mathrm{Cu}$ or $\mathrm{Ag}$; $\mathrm{B}^{\mathrm{II}}$ is from 
$\mathrm{Mn}, \mathrm{Fe}, \mathrm{Ni}, \mathrm{Zn}$ and $\mathrm{Cd}$; $\mathrm{C}^{\mathrm{IV}}$ is from IV main group elements $\mathrm{Si}, \mathrm{Ge}$ and $\mathrm{Sn}$; and $\mathrm{D}^{\mathrm{VI}}$ is from VI main group elements $\mathrm{S}$, Se and Te. They are originated from tetrahedrally coordinated derivatives of $\mathrm{B}^{\mathrm{II}}-\mathrm{D}^{\mathrm{VI}}$ binaries and have mostly diamond-like crystal structures [50]. These materials have recently received a great deal of attention due to their potential applications in thermoelectrics [51], spintronics [52], non-linear optics [53] and photocatalysts [54], and, thus, there has been a notable increase in their syntheses during the past few years.

Among this family of compounds, $\mathrm{Cu}_{2} \mathrm{FeSnS}_{4}$ has been experimentally synthesized $[55,56]$ and it manifests two tetragonal structure phases of the stannite type with space group $I \overline{4} 2 \mathrm{~m}$ and the quasi-cubic type with space group $P \overline{4}$. The two structures are very similar but assigned to different space groups because of the modified distributions of the containing metal elements. Although their magnetic susceptibility and optoelectronic properties have been investigated, a basic understanding on their the electronic, magnetic and mechanical properties is still missing. For this reason, we took the first quasi-cubic structure as an example and performed a systematic study with first principles calculation. The presence of half-metallicity in $\mathrm{Cu}_{2} \mathrm{FeSnS}_{4}$ was confirmed by its spin polarized band structures and the total magnetic moment of $4 \mu_{\mathrm{B}}$ was calculated with the main contribution from the $3 d$ transition metal $\mathrm{Fe}$ atoms. In addition, its mechanical properties were assessed and the corresponding spatial Young's modulus and shear modulus are obtained. Furthermore, the tetragonal strain conditions were also examined and their effects on the half-metallic and magnetic properties evaluated. It was found the total magnetic moment had almost no change, yet the half-metallicity could only be maintained under relatively small variations for both strains. This detailed work can provide a valuable reference regarding to the various properties for $\mathrm{Cu}_{2} \mathrm{FeSnS}_{4}$ and serve as a helpful guide for its future applications.

\section{Computational Methodology}

The electronic, magnetic and mechanical properties of the quaternary compound $\mathrm{Cu}_{2} \mathrm{FeSnS}_{4}$ were investigated with first principle calculations, which were performed with the pseudopotential plane wave methods [57] based on density functional theory (DFT) [58], using CASTEP package [59]. The generalized gradient approximation (GGA) [60] in the framework of the Perdew-Burke-Ernzerhof (PBE) functional [57] was adopted for the electronic exchange correlation energy. The valence electron configurations for $\mathrm{Cu}, \mathrm{Fe}, \mathrm{Sn}$ and $\mathrm{S}$ were treated as $3 \mathrm{~d}^{10} 4 \mathrm{~s}^{1}, 3 \mathrm{~d}^{6} 4 \mathrm{~s}^{2}, 5 \mathrm{~s}^{2} 5 \mathrm{p}^{2}$ and $3 \mathrm{~s}^{2} 3 \mathrm{p} 4^{2}$, respectively, and their interactions with the atomic core are described by the ultrasoft pseudopotential. Based on an initial convergence test, a cutoff energy of $500 \mathrm{eV}$ was set for the plane wave and a $\Gamma$ centered $10 \times 10 \times 10$ Monkhorst-Pack grid was selected for Brillouin zone $k$ point sampling. The crystal structure was fully relaxed until the total force per atom was smaller than $1 \times 10^{-3} \mathrm{eV} / \AA$. The self-consistent filed convergence tolerance was set as the total energy difference per atom less than $1 \times 10^{-6} \mathrm{eV}$.

\section{Results and Discussions}

The bulk quaternary compound $\mathrm{Cu}_{2} \mathrm{FeSnS}_{4}$ was experimentally synthesized and crystallized with a tetragonal symmetry; space group $P \overline{4}\left(S_{4}^{1}\right.$, No. 81$)$. Its crystal structure is schematically illustrated in Figure 1 and the unit cell contained eight atoms in total, including one Sn located at Wyckoff site 1a $(0,0,0)$, two Cu at Wyckoff site $2 \mathrm{~g}(0,0.5,0.5)$, four $\mathrm{S}$ at Wyckoff site $4 \mathrm{~h}(0.255,0.255,0.255)$ and one Fe at Wyckoff site 1c $(0.5,0.5,0)$. In this structure, each $S$ anion was coordinated with four cations and they formed slightly distorted tetrahedrons, as shown by the yellow shaded areas in Figure 1. The fully relaxed lattice constants for $\mathrm{Cu}_{2} \mathrm{FeSnS}_{4}$ were $a=5.416 \AA$ and $c=5.419 \AA$, which were consistent with the experimental values very well ( $a=5.4186 \AA$ and $c=5.4202 \AA$ ) [55]. These optimized lattice constants were used for the following discussions. 


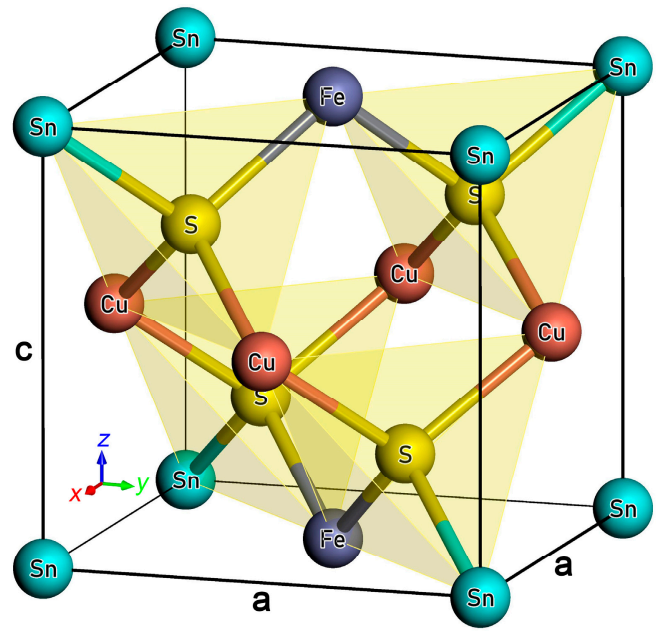

Figure 1. The crystal structures of the quaternary compound $\mathrm{Cu}_{2} \mathrm{FeSnS}_{4}$.

Based on the optimized crystal structure for $\mathrm{Cu}_{2} \mathrm{FeSnS}_{4}$, we calculated its electronic band structures. Since the $3 d$ transition metal element, Fe, carries a large magnetic moment, the band structures show spin polarized features (see Figure 2). The fermi energy is set to zero and only the energy range near the Fermi level is shown. In the spin-up direction, a small indirect band gap was observed with a conduction band minimum (CBM) located at high symmetry point $\mathrm{A}$ and the valence band maximum (VBM) at G. In the spin-down direction, two bands show multiple crossings with the Fermi energy level. This behavior of semiconductor properties in one spin direction and metal properties in the other spin direction clearly indicated that this material belongs to the family of half-metal compounds.
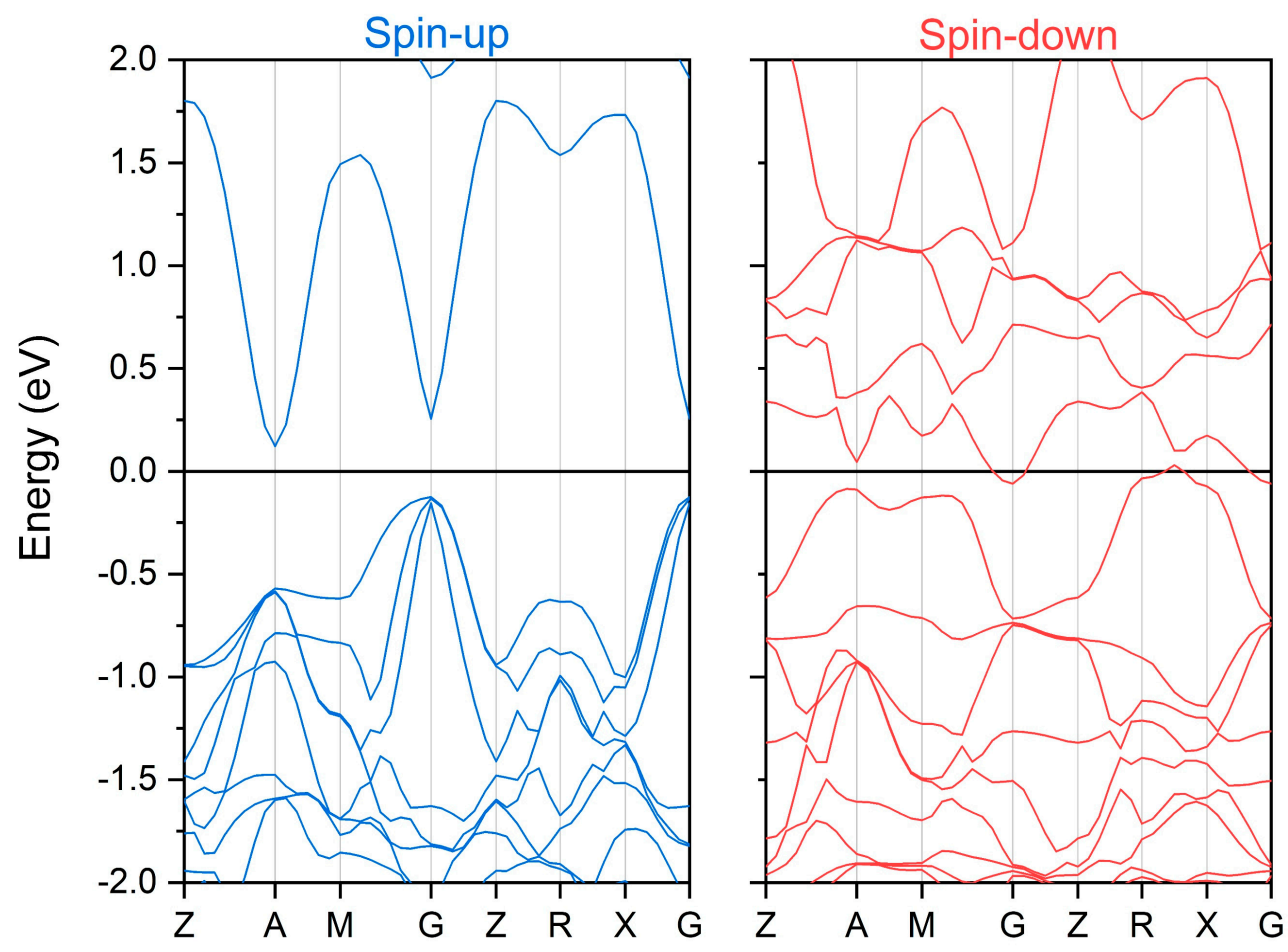

Figure 2. The calculated electronic band structure in both spin directions for $\mathrm{Cu}_{2} \mathrm{FeSnS}_{4}$ compound at the equilibrium lattice constants.

To further examine the correlation states between different elements and the magnetism origination for $\mathrm{Cu}_{2} \mathrm{FeSnS}_{4}$ compound, we have also computed the densities of states and the distributions of the magnetic spin density and the charge density difference, as reported in Figure 3. The positive and 
negative values in the total and partial densities of states in Figure 3a,b correspond to the spin-up and spin-down directions, respectively. A spin polarized behavior is observed: zero density at the Fermi level in spin-up direction and nonzero density in spin-down direction, which is in accordance with the findings from the electronic band structures. The total density of states near the Fermi level is mostly contributed from the transition metal elements $\mathrm{Fe}$ and $\mathrm{Cu}$, especially in the energy range from $-2 \mathrm{eV}$ to $+2 \mathrm{eV}$, and the main group elements, $\mathrm{Sn}$ and $\mathrm{S}$, have negligible contributions. However, the two transition metal elements exhibit different density distributions: an almost symmetric one for $\mathrm{Cu}$ element from $-2 \mathrm{eV}$ to $0 \mathrm{eV}$ between the two spin directions; a highly asymmetric one for $\mathrm{Fe}$ element with two high protrude areas in spin-up direction at -3 to $-2 \mathrm{eV}$; and several high peaks in spin-down direction at 0 to $1 \mathrm{eV}$. Especially for the density at the Fermi level in spin-down direction, it is completely from the Fe element. This strong spin splitting effect in the density of states for the Fe element could result in its strong magnetic moment, which indeed is confirmed by the calculated magnetic spin density as shown in Figure 3c. The large gray shaded areas around the Fe atoms indicate a strong local magnetic moment. Some shaded areas are also found around $\mathrm{Cu}$ atoms but they are much smaller compared with that around the Fe atoms. The calculated total magnetic moment per unit formula is $4 \mu_{\mathrm{B}}$ and it is mainly contributed by the Fe atom. The charge density difference is also computed to show the atomic bonding between different atoms and it is displayed in Figure $3 \mathrm{~d}$ with the gray shaded areas representing the electron accumulation. We can clearly see that the $S$ atoms form four bonds with the four neighbor atoms located at the corners of the slightly distorted tetrahedron and they have almost identical electron excess with only one a little bigger between $\mathrm{S}$ and Fe atoms. A very strong $d_{z^{2}}$ orbital shape is observed at the Fe atoms with the large dumbbell oriented to the center position.
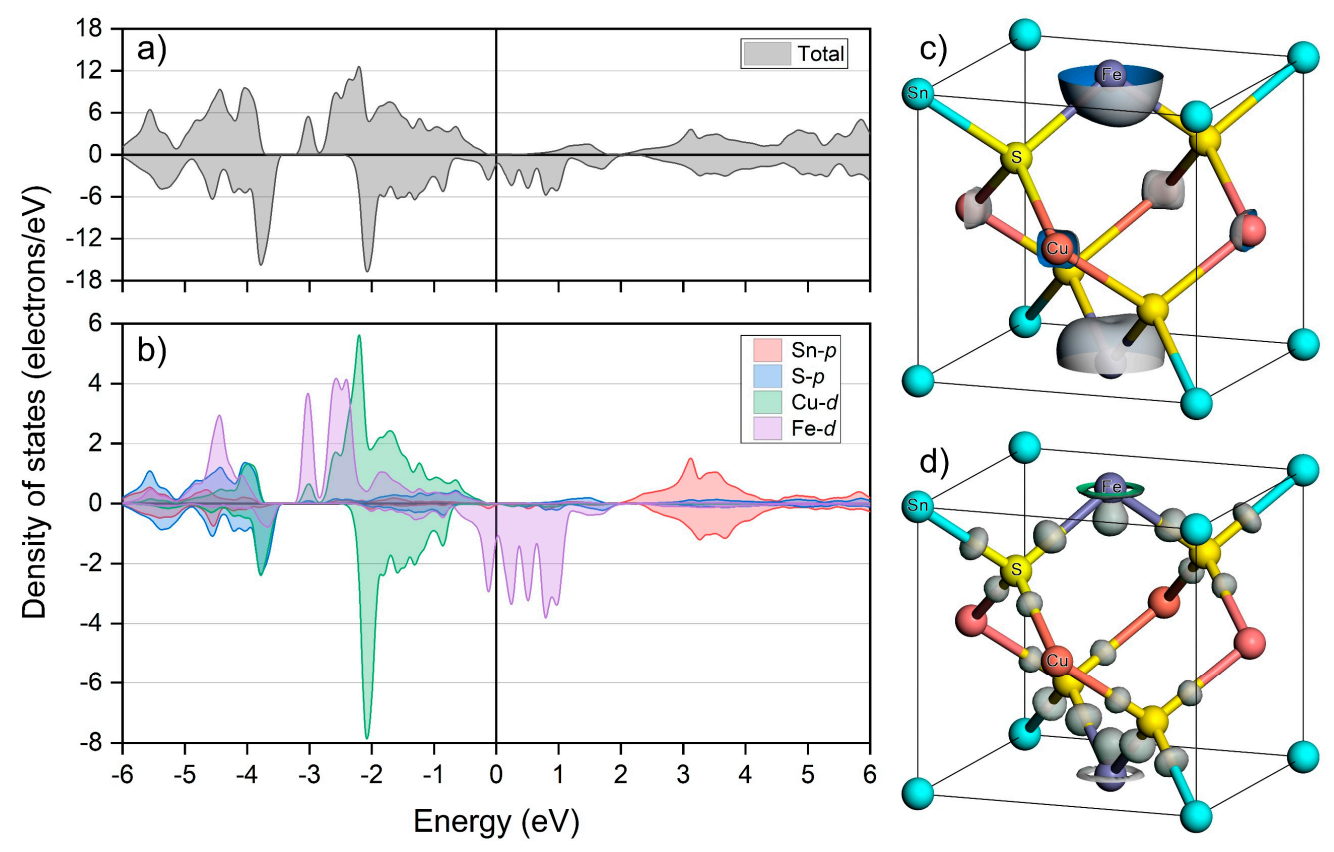

Figure 3. The calculated total (a) and partial (b) densities of states in both spin directions and the calculated magnetic spin density (c) and charge density difference (d) with isosurface value of 0.1 for $\mathrm{Cu}_{2} \mathrm{FeSnS}_{4}$ compound at the equilibrium lattice constants. The locations of different atoms in (c,d) can be referred to Figure 1.

Next, we turn to the mechanical properties of $\mathrm{Cu}_{2} \mathrm{FeSnS}_{4}$ compound. Since it has a $P \overline{4}$ tetragonal structure, there should be seven independent elastic constants in total: $C_{11}, C_{12}, C_{13}, C_{16}, C_{33}, C_{44}$ and $C_{66}$. By applying the stress-strain method [61], we successfully calculated their values, as summarized in Table 1. With these values, we can immediately examine the mechanical stability of $\mathrm{Cu}_{2} \mathrm{FeSnS}_{4}$ compound by employing the Born-Huang generalized elastic criteria [62], as follow: 


$$
\begin{gathered}
C_{11}>\left|C_{12}\right| \\
2 C_{13}^{2}>C_{33}\left(C_{11}+C_{12}\right) \\
C_{44}>0 \\
2 C_{16}^{2}>C_{66}\left(C_{11}-C_{12}\right)
\end{gathered}
$$

It is found that all conditions of Equation (1) are satisfied, verifying the mechanical stability of $\mathrm{Cu}_{2} \mathrm{FeSnS}_{4}$ compound. In addition, several other mechanical parameters can also be derived with the Voigt-Reuss-Hill approximation $[63,64]$, such as bulk modulus $B$, Young's modulus $E$, shear modulus $G$, compressibility $\beta$, Poisson's ratio $v$ and Pugh's ratio $(B / G)$, and their values are also included in Table 1. In general, the ductile/brittle behavior of material can also be referred to its Poisson's ratio $v$. For ductile materials, $v$ should be larger than $1 / 3$, otherwise the material behaves in a brittle manner. Here, the calculated $v$ of $\mathrm{Cu}_{2} \mathrm{FeSnS}_{4}$ is 0.314 , which is very close to the threshold value. Furthermore, Pugh's ratio $(B / G)$ can also be used to describe the brittle or ductile property of materials. When it is larger than 1.75, the material is ductile and vice versa. For $\mathrm{Cu}_{2} \mathrm{FeSnS}_{4}$ compound, its derived Pugh's ratio is 2.36, which is much larger than the threshold value. In combination, $\mathrm{Cu}_{2} \mathrm{FeSnS}_{4}$ is categorized as a ductile material.

Table 1. The calculated various elastic constants $\left(C_{\mathrm{ij}}\right)$, bulk modulus $B$, Young's modulus $E$, shear modulus $G$, compressibility $\beta$, Poisson's ratio $v$ and Pugh's ratio $(B / G)$ for $\mathrm{Cu}_{2} \mathrm{FeSnS}_{4}$.

\begin{tabular}{ccccccccccccc}
\hline $\begin{array}{c}C_{11} \\
(\mathrm{GPa})\end{array}$ & $\begin{array}{c}C_{12} \\
(\mathrm{GPa})\end{array}$ & $\begin{array}{c}C_{13} \\
(\mathrm{GPa})\end{array}$ & $\begin{array}{c}C_{16} \\
(\mathrm{GPa})\end{array}$ & $\begin{array}{c}C_{\mathbf{3 3}} \\
(\mathrm{GPa})\end{array}$ & $\begin{array}{c}C_{44} \\
(\mathrm{GPa})\end{array}$ & $\begin{array}{c}C_{66} \\
(\mathrm{GPa})\end{array}$ & $\begin{array}{c}B \\
(\mathrm{GPa})\end{array}$ & $\begin{array}{c}E \\
(\mathrm{GPa})\end{array}$ & $\begin{array}{c}G \\
(\mathrm{GPa})\end{array}$ & $\begin{array}{c}\beta \\
\left(\mathrm{TPa}^{-1}\right)\end{array}$ & $\boldsymbol{v}$ & $\boldsymbol{B} / \mathbf{G}$ \\
\hline 69.12 & 38.84 & 33.55 & 0.16 & 72.69 & 21.95 & 21.86 & 46.98 & 52.41 & 19.94 & 7.02 & 0.314 & 2.36 \\
\hline
\end{tabular}

Furthermore, the mechanical anisotropy is another very important property for the practical applications of materials, such as the epitaxial thin film growth and the heterojunction combination, and it is also accessed for $\mathrm{Cu}_{2} \mathrm{FeSnS}_{4}$ compound by calculating the spatial elastic moduli $[65,66]$. The directional dependent Young's modulus and shear modulus are shown in Figures 4 and 5, respectively. The $3 \mathrm{D}$ contour plot is accompanied with the $2 \mathrm{D}$ projections in different planes. It is found that the Young's modulus exhibits relatively small anisotropy because its spatial shapes are quite closed to the ideal isotropic sphere, yet the shear modulus shows strong directional anisotropy. This mechanical anisotropy is related with the crystal symmetry. For example, the Young's modulus exhibits fourfold central rotoinversion symmetry simply because the crystal system has the same symmetry operation. It should be noted here that although $\mathrm{Cu}_{2} \mathrm{FeSnS}_{4}$ compound has been synthesized in the experiment, there are still no direct mechanical parameters reported yet. Thus, the mechanical properties obtained in current work can provide a very helpful reference for its further study.
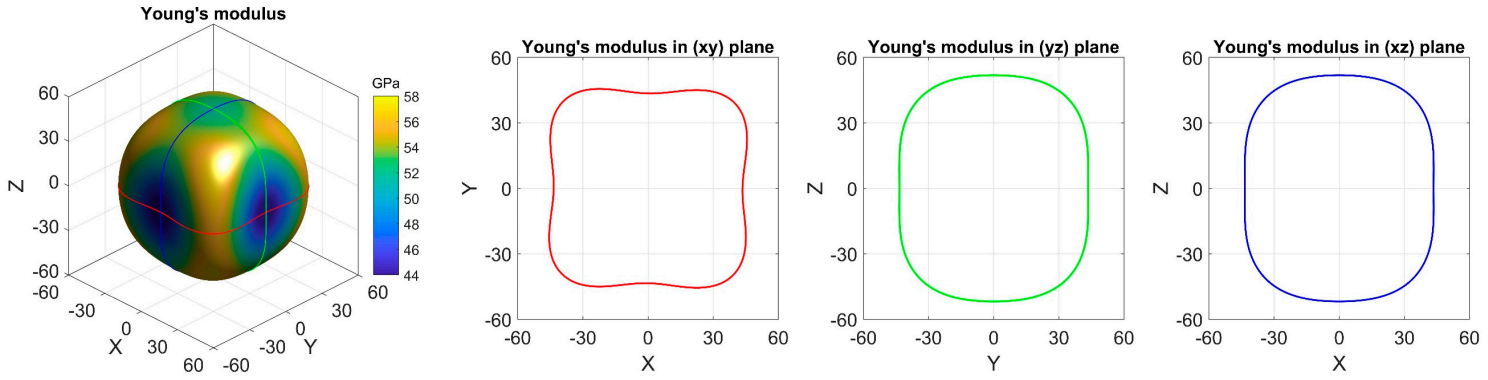

Figure 4. The calculated spatial Young's modulus for $\mathrm{Cu}_{2} \mathrm{FeSnS}_{4}$ compound at the equilibrium lattice constants. 

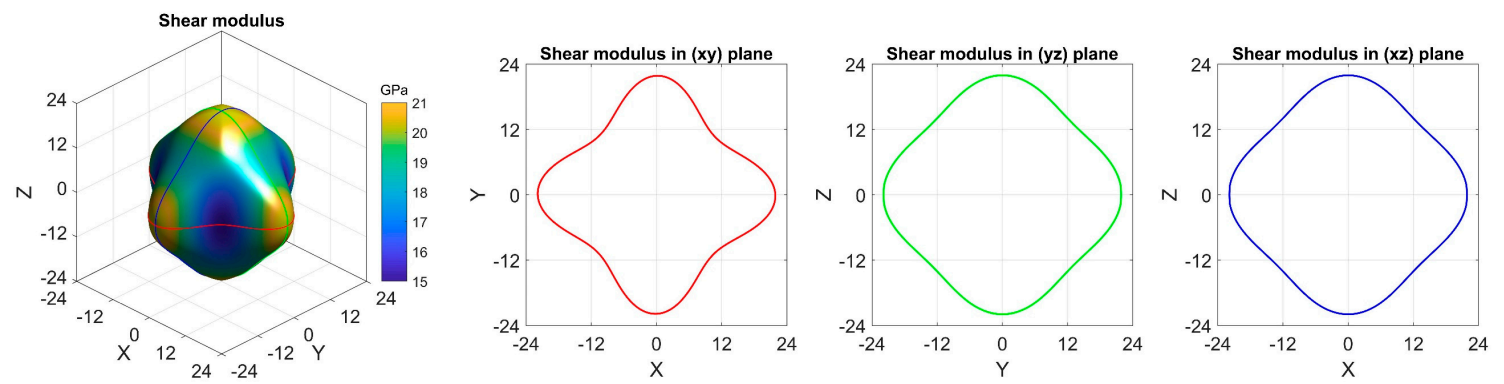

Figure 5. The calculated spatial shear modulus for $\mathrm{Cu}_{2} \mathrm{FeSnS}_{4}$ compound at the equilibrium lattice constants.

As is commonly known, a crystal structure cannot maintain an ideal equilibrium lattice in practical application and strain conditions often happen, especially during material processing and different structure connections in device construction. For some cases, lattice strain is also used on purpose to engineer materials' physical properties [67-70]. Consequently, we have also investigated the strain effects of $\mathrm{Cu}_{2} \mathrm{FeSnS}_{4}$. As this material has a tetragonal structure, there are two independent lattice constants, namely $a$ and $c$, and they are oriented perpendicular to each other. Thus, we introduced lattice strain in a tetragonal manner, that is to say, one lattice is varied when the other is kept constant. The considered lattice variation range is $\pm 5 \%$ from the equilibrium value for both two lattice constants.

Firstly, the total energy difference under different tetragonal strains was calculated and is plotted in Figure 6. Three green curves for the strain $a$ condition and three blue curves for the strain $c$ condition are shown, and they are perpendicularly orientated. In addition, two biaxial strain curves with black color are considered and they correspond to the positive biaxial strain condition with the same variation between $a$ and $c$ and the negative biaxial strain condition with the opposite variation between $a$ and $c$. It can be clearly observed from the figure that all strains lead to a total energy increase, implying that strain distortion makes $\mathrm{Cu}_{2} \mathrm{FeSnS}_{4}$ energetically unstable. Different variations are found: a much smaller total energy change with strain $c$ at zero strain $a$ compared with that with strain $a$ at zero strain $c$, see the central two perpendicular curves in Figure 6. This can be simply understood as follows: at the same strain ratio, the volume of the $\mathrm{Cu}_{2} \mathrm{FeSnS}_{4}$ crystal structure is varied linearly with strain $c$ but quadratically with strain $a$. It was also found that the reverse biaxial strain gave rise to a much smaller energy increase than the same biaxial strain, meaning the total energy variation of $\mathrm{Cu}_{2} \mathrm{FeSnS}_{4}$ compound was strongly correlated with its volume change.

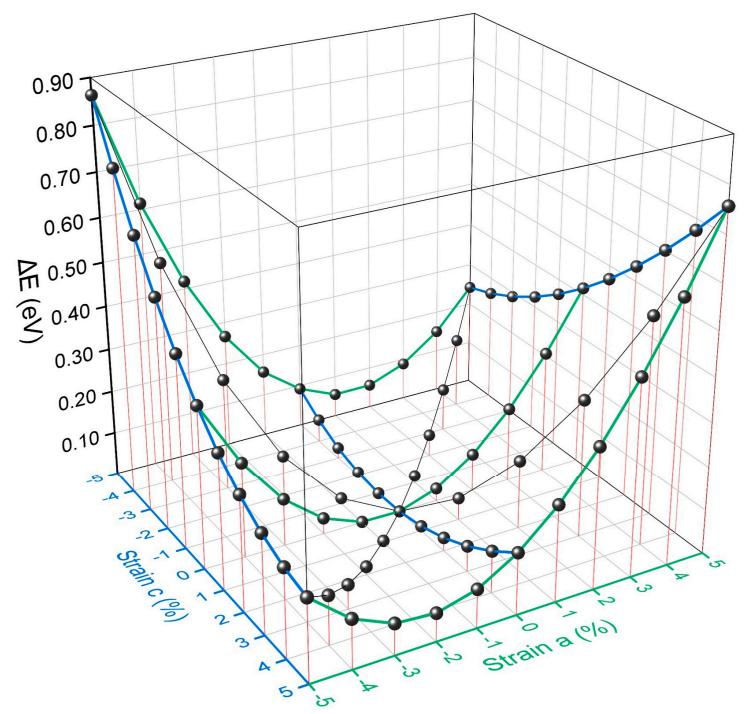

Figure 6. The calculated total energy difference with respect to the equilibrium condition for $\mathrm{Cu}_{2} \mathrm{FeSnS}_{4}$ compound under different tetragonal strains. 
Then, we moved on to examine the strain effects on the electronic properties for $\mathrm{Cu}_{2} \mathrm{FeSnS}_{4}$ compound. Since it shows half-metallic band structure with a small gap in the spin-up direction, we focused on the variations of CBM and VBM in the same spin under different tetragonal strains. The obtained results are reported in Figure 7, where the half-transparent gray area indicated the presence of band gap. We can see that CBM and VBM always descended with the strain increase from $-5 \%$ to $+5 \%$ for both $a$ and $c$. The band gap can be preserved under a much larger variation of strain $c$ at zero strain $a$ than strain $a$ at zero strain $c$, see the two plots in the central column. At $105 \%$ strain $a$, the band gap completely disappeared throughout the entire variation of strain $c$, which led to a total transformation from half-metal to metal.
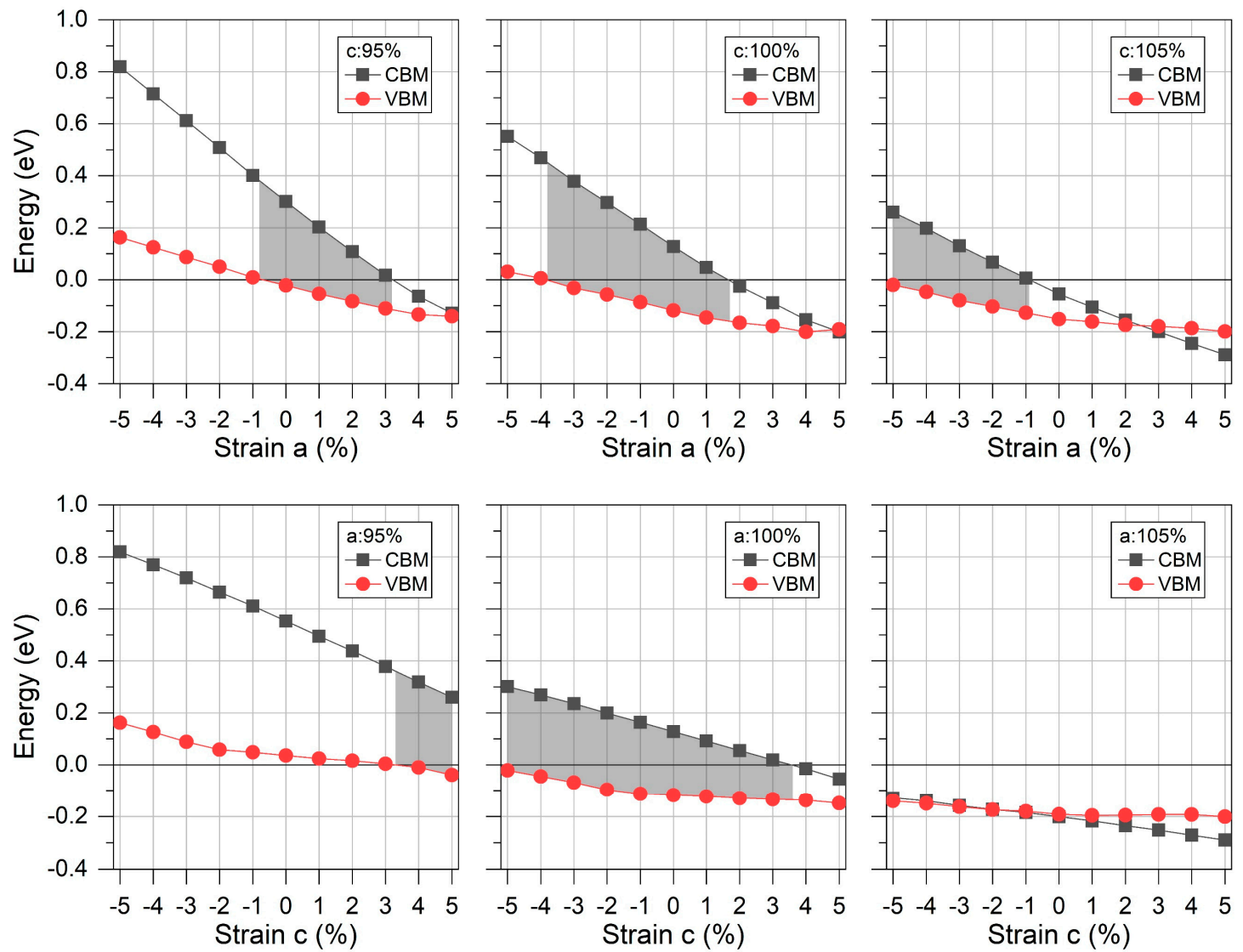

Figure 7. The calculated conduction band minimum (CBM) and valence band maximum (VBM) in the spin-up direction for $\mathrm{Cu}_{2} \mathrm{FeSnS}_{4}$ compound under different uniform strains. Grey shade indicates the area with band gap.

Finally, the total and atom-resolved partial magnetic moments were investigated under different tetragonal strains and their variations are shown in Figure 8. Because the total moment is mainly contributed to by the Fe atoms, different scales were applied in the vertical axis to have a better visualization. It is shown that the total magnetic moment was almost maintained constant over all strain variations, except for a very small drop at the compressive sides for both $a$ and $c$ of $95 \%$ strains, see the two plots in the left column. This unchanged total moment was due to the reverse variations between the Fe atoms and the other three atoms. The partial magnetic moment of the Fe atoms shows a relatively larger variation with strain $a$ than strain $c$, and its variation with strain $a$ (or strain $c$ ) only manifests a small offset between different $\operatorname{strain} c$ (or strain $a$ ) ratios. Overall, we found that the tetragonal strain has very small impact on the magnetic properties of $\mathrm{Cu}_{2} \mathrm{FeSnS}_{4}$. 

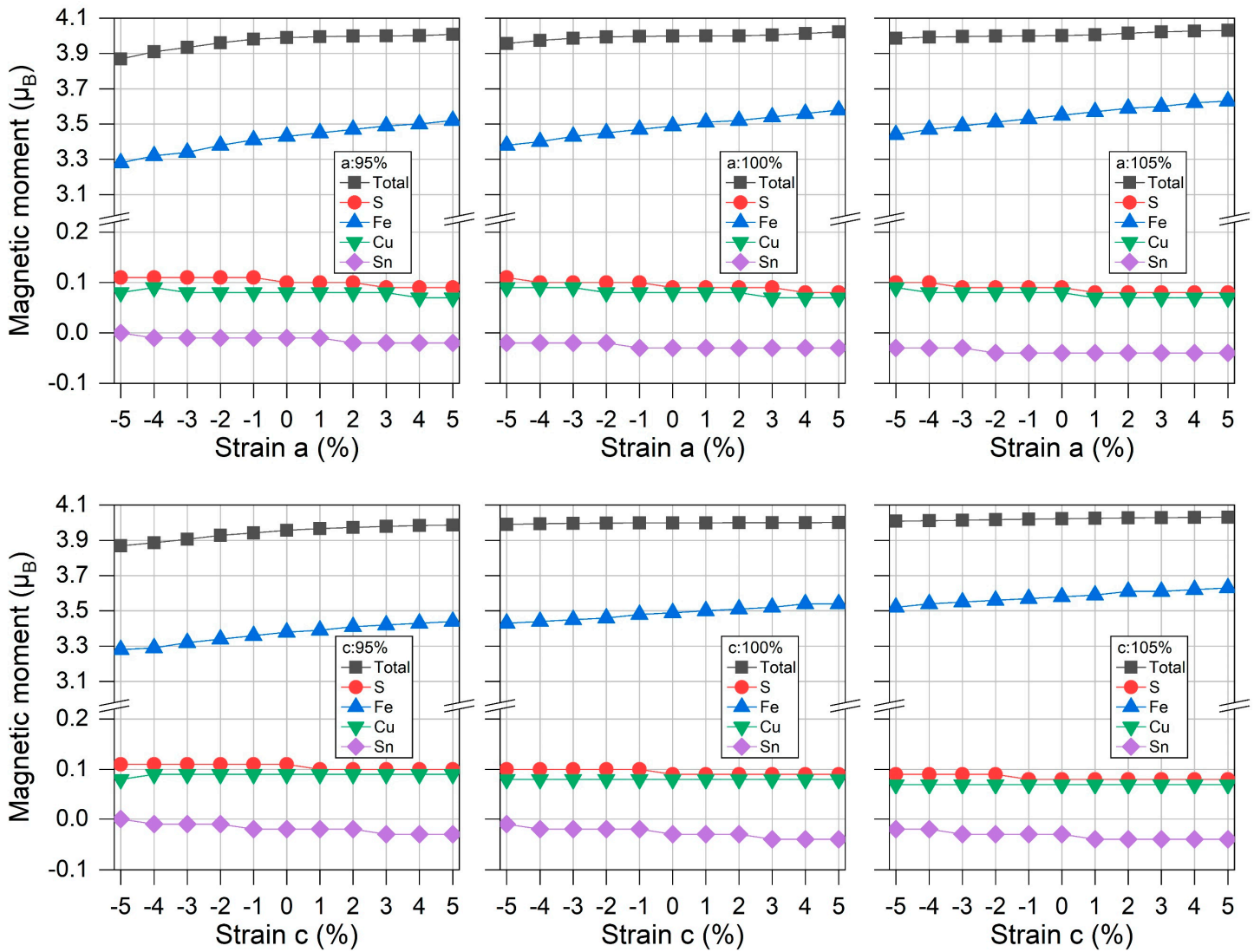

Figure 8. The calculated total and partial magnetic moments for $\mathrm{Cu}_{2} \mathrm{FeSnS}_{4}$ compound under different tetragonal strains. Note the different scales are used on the two sides of the break position in the vertical axis.

\section{Conclusions}

In conclusion, we have performed a systematic investigation of the electronic, magnetic and mechanical properties of the quaternary compound $\mathrm{Cu}_{2} \mathrm{FeSnS}_{4}$ using first principles calculations. The fully optimized lattice structure shows very good consistency with the corresponding experimental one. The spin polarized band structures show half-metallic behavior with a small band gap in the spin-up direction and multiple bands crossing the Fermi level in the spin-down direction. The calculated total magnetic moment is $4 \mu_{\mathrm{B}}$ and it is mostly contributed to by the $3 d$ transition metal element $\mathrm{Fe}$, which originated from its strong spin splitting densities of states and further confirmed by the large distribution of the magnetic spin density around the Fe atoms. Moreover, the mechanical properties were further studied and several mechanical parameters were obtained, with which the mechanical stability was verified. The spatial distribution of its Young's modulus and shear modulus were computed and it showed a very minor mechanical anisotropy. Finally, the tetragonal strain conditions have been further accessed and its effects on the electronic and magnetic properties for $\mathrm{Cu}_{2} \mathrm{FeSnS} 4$ compound have been examined. Results show the total magnetic moment can be properly maintained throughout the whole variation range for both strain $a$ and $c$, but the half-metallicity can only be preserved under relatively small variations. The current work can provide comprehensive information for quaternary compound $\mathrm{Cu}_{2} \mathrm{FeSnS}_{4}$ and a valuable reference for its future applications.

Author Contributions: Formal analysis, J.B., J.J. and L.H.; investigation, J.B., J.J. and L.H.; methodology, X.T.; project administration, L.H.; supervision, X.T.; validation, T.Y.; visualization, T.Y.; writing-original draft, T.Y. and X.T.; writing - review and editing, X.T. All authors have read and agreed to the published version of the manuscript.

Funding: This research received no external funding.

Conflicts of Interest: The authors declare no conflicts of interest. 


\section{References}

1. De Groot, R.A.; Mueller, F.M.; Engen, P.G.; Buschow, K.H.J. New Class of Materials: Half-Metallic Ferromagnets. Phys. Rev. Lett. 1983, 50, 2024-2027. [CrossRef]

2. Zutic, I.; Fabian, J.; Das Sarma, S. Spintronics: Fundamentals and applications. Rev. Mod. Phys. 2004, 76, 323. [CrossRef]

3. Gao, G.Y.; Yao, K.L.; Sasioglu, E.; Sandratskii, L.M.; Liu, Z.L.; Jiang, J.L. Half-metallic ferromagnetism in zinc-blende $\mathrm{CaC}, \mathrm{SrC}$, and $\mathrm{BaC}$ from first principles. Phys. Rev. B 2007, 75, 174442. [CrossRef]

4. Ishizuka, H.; Motome, Y. Dirac Half-Metal in a Triangular Ferrimagnet. Phys. Rev. Lett. 2012, 109, 237207. [CrossRef] [PubMed]

5. Li, X.; Yang, J. First-principles design of spintronics materials. Natl. Sci. Rev. 2016, 3, 365-381. [CrossRef]

6. Chadov, S.; Wu, S.-C.; Felser, C.; Galanakis, I. Stability of Weyl points in magnetic half-metallic Heusler compounds. Phys. Rev. B 2017, 96, 024435. [CrossRef]

7. Chen, X.; Huang, Y.; Yuan, H.; Liu, J.; Chen, H. Theoretical investigation on thermoelectric properties of spin gapless semiconductor Cr2ZnSi. Appl. Phys. A 2018, 124, 841. [CrossRef]

8. Patel, P.D.; Shinde, S.M.; Gupta, S.D.; Jha, P.K. A promising thermoelectric response of fully compensated ferrimagnetic spin gapless semiconducting Heusler alloy Zr2MnAl at high temperature: DFT study. Mater. Res. Express 2019, 6, 076307. [CrossRef]

9. Hasan, R.; Ur, S. Thermoelectric and Transport Properties of FeV1-xTixSb Half-Heusler System Synthesized by Controlled Mechanical Alloying Process. Electron. Mater. Lett. 2018, 14, 725-732. [CrossRef]

10. Kara, H.; Kahaly, M.U.; Ozdogan, K. Thermoelectric response of quaternary Heusler compound CrVNbZn. J. Alloys. Compd. 2018, 735, 950-958. [CrossRef]

11. Shigeta, I.; Kubota, T.; Sakuraba, Y.; Kimura, S.; Awaji, S.; Takanashi, K.; Hiroi, M. Transport properties of epitaxial films for superconductor $\mathrm{NbN}$ and half-metallic Heusler alloy Co2MnSi under high magnetic fields. Phys. B. Condens. Matter 2018, 536, 310-313. [CrossRef]

12. Nakajima, Y.; Hu, R.; Kirshenbaum, K.; Hughes, A.; Syers, P.; Wang, X.; Wang, K.; Wang, R.; Saha, S.R.; Pratt, D.; et al. Topological RPdBi half-Heusler semimetals: A new family of noncentrosymmetric magnetic superconductors. Sci. Adv. 2015, 1, e1500242. [CrossRef] [PubMed]

13. Sprungmann, D.; Westerholt, K.; Zabel, H.; Weides, M.; Kohlstedt, H. Evidence for triplet superconductivity in Josephson junctions with barriers of the ferromagnetic Heusler alloy Cu2MnAl. Phys. Rev. B 2010, 82, 060505. [CrossRef]

14. He, M.; Sun, H.; He, Q.L. Topological insulator: Spintronics and quantum computations. Front. Phys. 2019, 14, 43401. [CrossRef]

15. Chadov, S.; Qi, X.; Kubler, J.; Fecher, G.H.; Felser, C.; Zhang, S.C. Tunable multifunctional topological insulators in ternary Heusler compounds. Nat. Mater. 2010, 9, 541-545. [CrossRef] [PubMed]

16. Kainuma, R.; Imano, Y.; Ito, W.; Sutou, Y.; Morito, H.; Okamoto, S.; Kitakami, O.; Oikawa, K.; Fujita, A.; Kanomata, T.; et al. Magnetic-field-induced shape recovery by reverse phase transformation. Nature 2006, 439, 957-960. [CrossRef]

17. Zhu, W.; Liu, E.K.; Feng, L.; Tang, X.D.; Chen, J.L.; Wu, G.H.; Liu, H.Y.; Meng, F.B.; Luo, H.Z. Magnetic-fieldinduced transformation in FeMnGa alloys. Appl. Phys. Lett. 2009, 95, 222512. [CrossRef]

18. Planes, A.; Mañosa, L.; Acet, M. Magnetocaloric effect and its relation to shape-memory properties in ferromagnetic Heusler alloys. J. Phys. Condens. Matter 2009, 21, 233201. [CrossRef]

19. Wang, J.; Sehitoglu, H. Twinning stress in shape memory alloys: Theory and experiments. Acta Mater. 2013, 61, 6790-6801. [CrossRef]

20. Aznar, A.; Gràcia-Condal, A.; Planes, A.; Lloveras, P.; Barrio, M.; Tamarit, J.-L.; Xiong, W.; Cong, D.; Popescu, C.; Mañosa, L. Giant barocaloric effect in all-d-metal Heusler shape memory alloys. Phys. Rev. Mater. 2019, 3, 044406. [CrossRef]

21. Zeng, Q.; Shen, J.; Zhang, H.; Chen, J.; Ding, B.; Xi, X.; Liu, E.; Wang, W.; Wu, G. Electronic behaviors during martensitic transformations in all-d-metal Heusler alloys. J. Phys. Condens. Matter 2019, 31, 425401. [CrossRef] [PubMed]

22. Wang, X.; Khachai, H.; Khenata, R.; Yuan, H.; Wang, L.; Wang, W.; Bouhemadou, A.; Hao, L.; Dai, X.; Guo, R.; et al. Structural, electronic, magnetic, half-metallic, mechanical, and thermodynamic properties of the quaternary Heusler compound FeCrRuSi: A first-principles study. Sci. Rep. 2017, 7, 16183. [CrossRef] [PubMed] 
23. Cherid, S.; Benstaali, W.; Abbad, A.; Bentata, S.; Lantri, T.; Abbar, B. Theoretical prediction of half metallic ferromagnetic full-Heusler alloys Cs2CrGe. Solid State Commun. 2017, 260, 14-18. [CrossRef]

24. Bahramian, S.; Ahmadian, F. Half-metallicity and magnetism of quaternary Heusler compounds CoRuTiZ (Z=Si, Ge, and Sn). J. Magn. Magn. Mater. 2017, 424, 122-129. [CrossRef]

25. Han, Y.; Wu, Y.; Li, T.; Khenata, R.; Yang, T.; Wang, X. Electronic, Magnetic, Half-Metallic, and Mechanical Properties of a New Equiatomic Quaternary Heusler Compound YRhTiGe: A First-Principles Study. Materials (Basel) 2018, 11, 797. [CrossRef]

26. Zheng, A.M.; Huang, H.; Gao, G.Y.; Yao, K.L. Surface half-metallicity in the Heusler alloy Cr2CoGa with low magnetic moment. J. Mater. Sci. 2018, 53, 8364-8371. [CrossRef]

27. Hyun, J.M.; Kim, M. The Half-Metallicity of Co2FeGe Full Heusler Alloy in (001) Thin Film: First Principles Study. J. Korean Phys. Soc. 2018, 72, 276-282. [CrossRef]

28. Wang, J.X.; Chen, Z.B.; Gao, Y.C. Phase stability, magnetic, electronic, half-metallic and mechanical properties of a new equiatomic quaternary Heusler compound ZrRhTiIn: A first-principles investigation. J. Phys. Chem. Solids 2018, 116, 72-78. [CrossRef]

29. Wang, X.; Zhao, W.; Cheng, Z.; Dai, X.; Khenata, R. Electronic, magnetic, half-metallic and mechanical properties of a new quaternary Heusler compound ZrRhTiTl: Insights from first-principles studies. Solid State Commun. 2018, 269, 125-130. [CrossRef]

30. Liu, W.; Zhang, X.; Jia, H.; Khenata, R.; Dai, X.; Liu, G. Theoretical Investigations on the Mechanical, Magneto-Electronic Properties and Half-Metallic Characteristics of ZrRhTiZ (Z = Al, Ga) Quaternary Heusler Compounds. Appl. Sci. 2019, 9, 883. [CrossRef]

31. Chen, Y.; Chen, S.; Wang, B.; Wu, B.; Huang, H.; Qin, X.; Li, D.; Yan, W. Half-Metallicity and Magnetism of the Quaternary Heusler Compound TiZrCoIn1-xGex from the First-Principles Calculations. Appl. Sci. 2019, 9, 620. [CrossRef]

32. Ganai, Z.S.; Yousuf, S.; Batoo, K.M.; Khan, M.; Gupta, D.C. Half-metallicity and onsite Hubbard interaction on d-electronic states: A case study of Fe2NiZ (Z=Al, Ga, Si, Ge) Heusler systems. Philos. Mag. 2019, 99, 1551-1562. [CrossRef]

33. Rani, D.; Bainsla, L.; Suresh, K.G.; Alam, A. Experimental and theoretical investigation on the possible half-metallic behaviour of equiatomic quaternary Heusler alloys: CoRuMnGe and CoRuVZ ( $\mathrm{Z}=\mathrm{Al}, \mathrm{Ga})$. J. Magn. Magn. Mater. 2019, 492, 165662. [CrossRef]

34. Galanakis, I.; Mavropoulos, P. Spin-polarization and electronic properties of half-metallic Heusler alloys calculated from first principles. J. Phys. Condens. Matter 2007, 19, 315213. [CrossRef]

35. Kudrnovsky, J.; Drchal, V.; Turek, I.; Weinberger, P. Electronic, magnetic, and transport properties and magnetic phase transition in quaternary $(\mathrm{Cu}, \mathrm{Ni}) \mathrm{MnSb}$ Heusler alloys. Phys. Rev. B 2008, 78, 054441. [CrossRef]

36. Dai, X.F.; Liu, G.D.; Fecher, G.H.; Felser, C.; Li, Y.X.; Liu, H.Y. New quarternary half metallic material CoFeMnSi. J. Appl. Phys. 2009, 105, 07E901. [CrossRef]

37. Dannenberg, A.; Siewert, M.; Gruner, M.E.; Wuttig, M.; Entel, P. Competing structural ordering tendencies in Heusler-type alloys with high Curie temperatures: Fe2CoGa1-xZnx studied by first-principles calculations. Phys. Rev. B 2010, 82, 214421. [CrossRef]

38. Gao, G.Y.; Hu, L.; Yao, K.L.; Luo, B.; Liu, N. Large half-metallic gaps in the quaternary Heusler alloys $\mathrm{CoFeCrZ} \mathrm{(Z=Al,} \mathrm{Si,} \mathrm{Ga,} \mathrm{Ge):} \mathrm{A} \mathrm{first-principles} \mathrm{study.} \mathrm{J.} \mathrm{Alloys.} \mathrm{Compd.} \mathrm{2013,} \mathrm{551,} \mathrm{539-543.} \mathrm{[CrossRef]}$

39. Özdoğan, K.; Şaşığlu, E.; Galanakis, I. Slater-Pauling behavior in LiMgPdSn-type multifunctional quaternary Heusler materials: Half-metallicity, spin-gapless and magnetic semiconductors. J. Appl. Phys. 2013, 113, 193903. [CrossRef]

40. Delgado, G.E.; Quintero, E.; Tovar, R.; Grima-Gallardo, P.; Quintero, M. Synthesis and crystal structure of the quaternary compound AgFe2GaTe4. J. Alloys. Compd. 2014, 613, 143-145. [CrossRef]

41. Berri, S.; Ibrir, M.; Maouche, D.; Attallah, M. First principles study of structural, electronic and magnetic properties of ZrFeTiAl, ZrFeTiSi, ZrFeTiGe and ZrNiTiAl. J. Magn. Magn. Mater. 2014, 371, 106-111. [CrossRef]

42. Berri, S.; Ibrir, M.; Maouche, D.; Attallah, M. Robust half-metallic ferromagnet of quaternary Heusler compounds ZrCoTiZ(Z = Si, Ge, Ga and Al). Comput. Condens. Matter 2014, 1, 26-31. [CrossRef]

43. Delgado, G.; Delgado, N.; Gallardo, P. Crystal structure of the quaternary semiconductor compound CuFeCrSe3. Period. Tche Quim. 2019, 16, 1 . 
44. Hao, L.; Tan, M.; Khenata, R.; Wang, X.; Yang, T. Computational study of the electronic, magnetic, mechanical and thermodynamic properties of new equiatomic quaternary heusler compounds TiZrRuZ (Z $=\mathrm{Al}, \mathrm{Ga}, \mathrm{In})$. Chin. J. Phys. 2019, 62, 54-64. [CrossRef]

45. Schäfer, W.; Nitsche, R. Tetrahedral quaternary chalcogenides of the type $\mathrm{Cu}_{2}$-II-IV-S $4\left(\mathrm{Se}_{4}\right)$. Mater. Res. Bull. 1974, 9, 645-654. [CrossRef]

46. Wintenberger, M. Etude de la structure cristallographique et magnetique de Cu2FeGeS4 et remarque sur la structure magnetique de Cu2MnSnS4. Mater. Res. Bull. 1979, 14, 1195-1202. [CrossRef]

47. Delgado, G.E.; Sierralta, N.; Quintero, M.; Quintero, E.; Moreno, E.; Flores-Cruz, J.A.; Rincon, C. Synthesis, structural characterization and differential thermal analysis of the quaternary compound $\mathrm{Ag}_{2} \mathrm{MnSnS}_{\text {. }}$. Rev. Mex. Fis. 2018, 64, 216-221. [CrossRef]

48. Delgado, G.; Sagredo, A. Synthesis and crystal structure of the quaternary semiconductor Cu2NiGeS4, a new stannite-type compound. Rev. Mex. Física 2019, 65, 355. [CrossRef]

49. Guen, L.; Glaunsinger, W.S. Electrical, magnetic, and EPR studies of the quaternary chalcogenides Cu2AIIBIVX4 prepared by iodine transport. J. Solid State Chem. 1980, 35, 10-21. [CrossRef]

50. Giriat, W.; Furdyna, J. Chapter 1 Crystal Structure, Composition, and Materials Preparation of Diluted Magnetic Semiconductors; Elsevier BV: Amsterdam, The Netherlands, 1988; Volume 25, pp. 1-34.

51. Grima-Gallardo, P.; Cárdenas, K.; Molina, L.; Quintero, M.; Ruiz, J.; Delgado, G.; Briceño, J.M. A Comparative Study of (Cu-III-Se2)x-(FeSe) $1-x$ Alloys (III: Al, Ga, In) $(0 \leq x \leq 1)$ by X-Ray Diffraction, Differential Thermal Analysis and Scanning Electron Microscopy. Phys. Status Solidi (a) 2001, 187, 395-406. [CrossRef]

52. Chambers, S.A.; Yoo, Y.K. New Materials for Spintronics. MRS Bull. 2003, 28, 706-710. [CrossRef]

53. Catella, G.C.; Burlage, D. Crystal Growth and Optical Properties of AgGaS2 and AgGaSe2. MRS Bull. 1998, 23, 28-36. [CrossRef]

54. Tsuji, I.; Shimodaira, Y.; Kato, H.; Kobayashi, H.; Kudo, A. Novel Stannite-type Complex Sulfide Photocatalysts $\mathrm{AI}-\mathrm{Zn}$-AIV-S4 (AI = $\mathrm{Cu}$ and $\mathrm{Ag} ; \mathrm{AIV}=\mathrm{Sn}$ and $\mathrm{Ge})$ for Hydrogen Evolution under Visible-Light Irradiation. Chem. Mater. 2010, 22, 1402-1409. [CrossRef]

55. Rincón, C.; Quintero, E.; Quintero, M.; Moreno, E.; Power, C.; Morocoima, M.; Delgado, G.E. Temperature Dependence of Raman Spectra in Cu2FeSnS4 Magnetic Semiconductor Compound. Phys. Status Solidi (b) 2019, 10, 1900076.

56. Rincón, C.; Quintero, M.; Moreno, E.; Power, C.; Quintero, E.; Henao, J.A.; Macías, M.A.; Delgado, G.E.; Tovar, R.; Morocoima, M. X-ray diffraction, Raman spectrum and magnetic susceptibility of the magnetic semiconductor Cu2FeSnS4. Solid State Commun. 2011, 151, 947-951. [CrossRef]

57. Vanderbilt, D. Soft self-consistent pseudopotentials in a generalized eigenvalue formalism. Phys. Rev. B. Condens. Matter. 1990, 41, 7892-7895. [CrossRef]

58. Payne, M.C.; Teter, M.P.; Allan, D.C.; Arias, T.A.; Joannopoulos, J.D. Iterative minimization techniques forab initiototal-energy calculations: Molecular dynamics and conjugate gradients. Rev. Mod. Phys. 1992, 64, 1045-1097. [CrossRef]

59. Segall, M.D.; Lindan, P.J.D.; Probert, M.J.; Pickard, C.J.; Hasnip, P.J.; Clark, S.J.; Payne, M.C. First-principles simulation: Ideas, illustrations and the CASTEP code. J. Phys. Condens. Matter 2002, 14, 2717-2744. [CrossRef]

60. Perdew, J.P.; Burke, K.; Ernzerhof, M. Generalized Gradient Approximation Made Simple. Phys. Rev. Lett. 1996, 77, 3865-3868. [CrossRef]

61. Wang, J.; Li, J.; Yip, S.; Phillpot, S.; Wolf, D. Mechanical instabilities of homogeneous crystals. Phys. Rev. B. Condens. Matter 1995, 52, 12627-12635. [CrossRef]

62. Mouhat, F.; Coudert, F.X. Necessary and sufficient elastic stability conditions in various crystal systems. Phys. Rev. B 2014, 90, 224104. [CrossRef]

63. Hill, R. The Elastic Behaviour of a Crystalline Aggregate. Proc. Phys. Soc. Sect. A 1952, 65, 349. [CrossRef]

64. Yip, S.; Li, J.; Tang, M.J.; Wang, J.G. Mechanistic aspects and atomic-level consequences of elastic instabilities in homogeneous crystals. Mater. Sci. Eng. A Struct. Mater. Prop. Microstruct. Process. 2001, 317, $236-240$. [CrossRef]

65. Gaillac, R.; Pullumbi, P.; Coudert, F.X. ELATE: An open-source online application for analysis and visualization of elastic tensors. J. Phys. Condens. Matter 2016, 28, 275201. [CrossRef] [PubMed]

66. Marmier, A.; Lethbridge, Z.A.D.; Walton, R.I.; Smith, C.W.; Parker, S.C.; Evans, K.E. ElAM: A computer program for the analysis and representation of anisotropic elastic properties. Comput. Phys. Commun. 2010, 181, 2102-2115. [CrossRef] 
67. Yang, T.; Hao, L.Y.; Khenata, R.; Wang, X.T. Strain Conditions for the Inverse Heusler Type Fully Compensated Spin-Gapless Semiconductor Ti2MnAl: A First-Principles Study. Materials 2018, 11, 2091. [CrossRef]

68. Yang, T.; Cao, J.; Khenata, R.; Cheng, Z.; Kuang, M.; Wang, X. Strain effect for the newly discovered spin-gapless diamond-like quaternary-type semiconductor CuMn2InSe4. J. Alloys. Compd. 2019, 793, 302-313. [CrossRef]

69. Yang, T.; Hao, L.; Khenata, R.; Wang, X. Investigation of the structural competing and atomic ordering in Heusler compounds Fe2NiSi and Ni2FeSi under strain condition. R. Soc. Open Sci. 2019, 6, 191007. [CrossRef]

70. You, J.; Cao, J.; Khenata, R.; Wang, X.; Shen, X.; Yang, T. Robust Spin-Gapless Behavior in the Newly Discovered Half Heusler Compound MnPK. Materials (Basel) 2019, 12, 3117. [CrossRef]

(C) 2020 by the authors. Licensee MDPI, Basel, Switzerland. This article is an open access article distributed under the terms and conditions of the Creative Commons Attribution (CC BY) license (http://creativecommons.org/licenses/by/4.0/). 